\title{
What Cannabis Can Do to a Man?: A Case Report
}

\author{
Dhana Ratna Shakya*, and Aparna Ghimire \\ Department of Psychiatry, BP Koirala Institute of Health Sciences, Dharan, Nepal
}

*Corresponding author: Dhana Ratna Shakya, Professor, Department of Psychiatry, BP Koirala Institute of Health Sciences, Dharan, Nepal, E-mail: drdhanashakya@yahoo.com

Received: 05 Jul, 2021 | Accepted: 19 Aug, 2021 | Published: 27 Aug, 2021

Citation: Shakya DR, Ghimire A (2021) What Cannabis Can Do to a Man?: A Case Report. J Psychiatry Ment Health 6(2): dx.doi. org/10.16966/2474-7769.144

Copyright: (C) 2021 Shakya DR, et al. This is an open-access article distributed under the terms of the Creative Commons Attribution License, which permits unrestricted use, distribution, and reproduction in any medium, provided the original author and source are credited.

\begin{abstract}
Many people use cannabis for various reasons, mainly recreational or medicinal purposes. Many of them start using it on the belief of or in the influence of attractive messages of benefit and positive effects. A common Nepalese citizen comes across conflicting messages and information regarding cannabis though the information about its harms and adverse effects is less highlighted and reaches less to related stakeholders. Amidst such a conflicting situation, a victim is bound to endure a cascade of suffering in many ways, as is explicit in the eyes of a mental health professional. We present here a case to demonstrate how serious diverse and what adverse effects cannabis use can bring to the person's health, life and family.
\end{abstract}

Keywords: Cannabis; Complications; Effects; Harms; Psychiatric co-morbidity

List of Abbreviations: CIPD: Cannabis Induced Psychotic Disorder; CDS: Cannabis Dependence Syndrome; NDS: Nicotine Dependence Syndrome; ED: Emergency Department; ICD: International Classification of Disease; WHO: World Health Organization

\section{Key Clinical Message}

We present a case of cannabis dependence; with: well adjusted temperament, average intellectual and over all functioning, sound physical health premorbidly; and no substance use and mental disorder in close family. He started cannabis use at age 18 with peer circle and over 8 years of its use suffered a series of complications and adversities consequent to its long and heavy use, including cannabis dependence, cannabis induced psychotic disorder (CIPD) (bipolar mood: mania during intoxication and depression during withdrawal), presented in catatonia requiring admission in Psychiatric ward.

\section{Introduction}

Cannabis, with active ingredient $\Delta-9$ tetrahydrocannabinol (THC) of plant Cannabis sativa is the most widely cultivated, trafficked and abused illicit psychoactive substance worldwide [1]. Proponents of cannabis take its use as a pleasant and non-threatening experience (recreational) and also beneficial for many health reasons (medicinal) [2]. Opponents, though less highlighted and hardly reach to the stakeholders' attention, point out its various adverse effects across the course of its use (acute and long term effects) [3]. Hence, the review of its harm and benefits has been an important prospect. A recent review pointed out that its medicinal properties, use and benefits are yet to undergo scientific research for evidence while harms do not lack evidence [4].

As mental health professionals, we intend here to present and discuss in brief the myriad of adverse consequences of long and heavy cannabis use in reference to our case who and whose family had to face in health and life. We can appreciate the implication of COVID-19 lockdown situation in the course of illness in this case, as in a case report recently published from the same setting [5].

\section{Case Presentation}

We present here a case of 26 year gentleman from Itahari, a city area of Sunsari district in eastern Nepal. He has been studying in bachelor level of civil engineering as major and is currently unemployed. He belongs to a joint family of middle socioeconomic status with farming and foreign remittance as major income sources. He started taking cannabis at the age of 18 years due to peer influence. He continued taking in sticks (2-3 sticks per day), gradually increasing the amount and frequency. By the time he was 21 years, he started taking in chilim or homemade water bong. He joined engineering at the age of 23 years when he started living by himself in Kathmandu, capital city of Nepal. The intake of cannabis increased so much so that he started consuming cannabis every half an hour. He used to take alcohol occasionally and cigarette daily. He experimented with benzodiazepine tablet, opioid tablet and brown sugar on separate occasions. But, he never continued their intake as they were not as readily available as cannabis. $\mathrm{He}$ skipped classes and even didn't appear in exams while enjoying the effects of cannabis. While joining engineering, he had dream of achieving something in this field because Nepal is a developing nation and there are so many possibilities for being a contributor in 
its development. Currently recalling the experience, he says he was not aware about the impact cannabis could have made in his life. At that time, he barely realized that it was not a desired behaviour because all his friends were down in the same path. He recalls one event of a bad road traffic accident while he and one of his friends were riding scooter under influence of cannabis. His friend broke his arm in the accident, but they realized this only after his friend rode for another 10-15 minutes with the same broken arm. He also recalls being warned by police personnel as he hadn't hesitated to smoke cannabis in the college premises itself.

After taking very high dose of cannabis for 2-3 years, family members began noticing some change in patient's behaviour. While under influence, he was talking more than usual, mostly about new and special ideas. He didn't sleep at night but started roaming around with new ideas and feeling of being able to do anything. He presently recalls that he had increased self esteem and had thoughts about being special. He couldn't be still even for few moments. There was lot of energy inside him. His life was so affected that he didn't care for eating or sleeping, studies were neglected for long time and every penny he had was invested in buying cannabis. His friend circle was limited to cannabis user, rest of the people seem to be avoiding him. While this went on for nearly 1 month, his family member started living with him and was actively trying to reduce the amount of his cannabis intake. So within next month, he became better. He had been taking 20 to 30 sticks of cannabis per day. He appeared his final exam with his mother staying with him.

Just before lockdown (in May 2021), they returned back home. Due to the effect of lockdown in response to COVID-19, he was not able to get as much cannabis as he was consuming previously. So, his dose decreased to 2-3 sticks per day. As he decreased his dose, he started feeling low, most of the time of the day. He didn't have any interest in interacting with anyone. He had no interest in doing anything. He was confined in his own room most of the time. He felt weak. He had decreased appetite and it was difficult to initiate sleep till 1-2 am in the morning. Now, he didn't even have any energy to search for cannabis. So, he stopped taking cannabis altogether. After 2 days of completely stopping cannabis, he was observed to have marked psychomotor retardation. He didn't speak at all. He refused food and water. Even when he was tried to be fed by family, he clenched his teeth and refused opening mouth. He was standing still in one posture for hours at a time. He didn't sleep. When these behaviours lasted for 2 days continuously, he was brought to emergency department (ED) of our institute [6] and was admitted for management purpose. Currently, patient recalls those two days as being confused inside and was unable to respond appropriately. He remembers thinking why it was happening, how it was happening and was perplexed.

Blood investigations ( $\mathrm{CBC}$, serum electrolytes, liver function test, blood glucose and renal function tests) were done. He was given intravenous benzodiazepine (diazepam) in view of catatonia. His psychomotor activity improved within few hours of medication further confirming the diagnosis. Impression of Cannabis dependence syndrome (CDS) with cannabis induced psychotic disorder (mania, during intoxication; depression, during withdrawal) (WHO-ICD-10 diagnosis) presented in catatonia with Nicotine dependence syndrome (NDS) and multiple substance use was made. CT scan of head was done to rule out any organic basis for catatonia which was normal. All other investigations were in normal limits. There was no family history of mental disorders except an uncle's suicide which seems secondary to a stressor and not with known mental illness.
During in-patient management, he was assessed for motivation for abstinence from cannabis. In first day, he was not aware about any role of cannabis in his present condition and hence didn't think he needed to quit. When the treating team presented the temporal relation of his symptom with cannabis and no other diagnosis could explain his presentation, he understood the effect of cannabis. As motivation enhancement assessment, he wrote what benefits he thought cannabis could do. He used to think that cannabis is medicinal, it makes us strong. But reflecting upon the impact cannabis had on own mental health, he later wrote that if there was no cannabis in his life, he wouldn't have needed admission in psychiatry ward. He also mentioned cannabis as attraction for tourist and way to develop country. But upon looking back his own life, he wrote if there was no cannabis, he could have completed his study to fulfill his dream. He also regretted about the time and money he spent on cannabis in all these years which could have been put into better use. He remembers all the friends he lost because of his substance taking behaviour who now didn't prefer his company. The irony here is that he is an undergraduate student with science as major subject, but yet, he was unaware about any consequences cannabis could have upon his physical or mental health. The patient was almost euthymic, with normal energy and confidence level after few days of hospitalization. On the $12^{\text {th }}$ day of admission, he was discharged upon request by his family member despite having mild restlessness and sense of apprehension because there were other new fever cases in ward in context of COVID-19 pandemic.

The patient came to our out-patient clinic within a week of discharge with complains of restlessness, sleep disturbance, decreased appetite and observed agitation. He was persistently sad. He had low energy level and low self esteem. He started having frequent ideas of hopelessness, helplessness and worthlessness. Antidepressant medication was started along with benzodiazepine in view of 'severe depressive episode with prominent anxiety features'. But, patient's symptom gradually worsened over next 2 weeks. His agitation was markedly increased. He had flight of ideas mostly with underlying depressive content. He also started having recurrent ideas of suicide and was expressing them to family members. There was one aborted suicide attempt. On the $3^{\text {rd }}$ week of discharge, he was readmitted. His medications were revised to mood-stabilizer, antipsychotic and anxiolytics. The ongoing in-patient treatment included encouragement in scheduled daily activity, prompt optimization of medications and daily monitoring with supportive approach. We still were struggling to bring improvement in his anxiety symptoms, low mood, low self esteem, ideas of helplessness, hopelessness, worthlessness and occasional death wishes in the $3^{\text {rd }}$ week of readmission though there was some improvement in sleep pattern and psychomotor agitation.

\section{Discussion}

Our patient is the youngest sibling and was loved by all his family members. He used to be well mannered youth, with uneventful childhood, no history of emotional trauma/abuse; with premorbid temperament as easy child; no disciplinary or behavioural issues during school life. His family of middle socioeconomic status and well educated parents provided him congenial environment for education. He had stable interpersonal relationship with others. He enjoyed a sound physical health. There was no history of known mental illness (except history of suicide in uncle which seems secondary to a stressor and no known mental illness) and substance abuse in family. Cannabis influence was so powerful among the youths in college that such a motivated, well mannered youth happened to start using cannabis in his peer circle at age of 18 years. 
He started having abdominal pain if cannabis was not available, he had loss of appetite and weight, he had sleep disturbance and he sustained a road traffic accident while riding scooter under the influence, to list some of physical complications. In such a young productive age, he had craving of cannabis, neglect of all other pleasurable activities even including study and exams of Engineering; most of his time was spent on acquiring or consuming cannabis and other substances; developed mania (irritability, boastful talk, talkativeness, special plan and idea, decreased need for sleep) while heavily consuming cannabis; developed depression (persistently low mood, decreased energy, loss of interest, loss of concentration, decreased sleep) after cutting down on cannabis; presented in catatonic state (mutism, posturing, staring, decreased psychomotor activity); sense of low self esteem; guilt feeling regarding the cannabis; wide range of serious psychological complications eventually requiring hospitalization in De-addiction/ Psychiatric ward, that too in lockdown time of COVID-19 pandemic [6]. He also used other substances; had nicotine dependence syndrome, use of opioid, benzodiazepine tablet and brown sugar; cannabis acting as 'gateway drug' to later ones. Medical literature reviews reveal such adverse psychological consequences, i.e. wide range of mental comorbidities including psychosis [3-8]. A recent review asserts that its harms and adverse effects are adequately evidence based, mainly from the perspective of mental health effects (psychosis, bipolar disorder, depression, anxiety etc.) [4]. He had high suicidality too.

The patient had behavioral changes; became more impulsive (having verbal altercations with other people around, out of anger outburst), he used to throw or break whatever thing he had in his hand. His interpersonal relation with close family members and his girl friend was strained; he had frequent conflict with girlfriend which was a constant stressor in the patient. He, at times when sober, realized that he had lost his family's trust upon him, felt like a burden upon the family and relationship with family member turned out poor. He felt that he was seen by the society with less respect (as a substance user), lost all the friends who didn't consume substance, and also stigma for being admitted in mental health facility. He had spent all the money he had on cannabis, never had economically productive life as his time and focus was preoccupied by cannabis intake and the cost of treatment/ transportation during lock down period and loss of wages of brother while needed to stay in hospital for him added to the burden. He had not been able to pass bachelor of engineering, had back paper in every year. He had not ever planned for any job yet, a great concern of his family including old parents for this grown up educated person. He had at times been warned by police for consuming cannabis in college premises too.

We can appreciate a wide range of psychosocial and legal problems surrounding this man as reported for the cannabis users long back by our first psychiatrist from Nepal [9].

\section{Conclusion}

Our case report highlights the fact that cannabis use can bring adverse consequences to the health and life of person and family, even including severe psychiatric condition of psychosis, bipolar mood and catatonia. Careful assessment of etiological (bio-psycho-social and HAVE model) [10] factors indicates the potentially useful and effective management strategies. Acute management needs to be complimented with Motivational intervention, and continued with rehabilitation efforts. Feedback to the complications of the cannabis and substance use, awareness and insight to them build the foundation of management of Cannabis use disorder as a psychoactive substance. As evident from this case report, cannabis has addictive and psychoactive property affecting both vulnerable people like those with mental illness and even healthy ones with long and heavy use as our case. Consequently, myriads of complications befall upon the consumer. Hence, the trend and step towards legalization of cannabis to open door for its industry efforts, i.e. its cultivation, research, development, advertising, and distribution (Vector factors) and more congenial environment or agreeing attitude of society [10] should be taken seriously and needs to be reviewed. COVID-19 pandemic has some local contextual implications in the course of cannabis/ substance use disorders which can be a subject of further study [5].

\section{Consent}

Informed consent was received from the patient.

\section{Author Contributions}

DRS- conception and design, collection of data, drafting and editing of the manuscript; AG- involved in collection of data, drafting of the manuscript and both the authors approval of final version.

\section{Ethical Approval}

Ethical approval was not required.

\section{Conflict of Interest}

The authors state that there is no conflict of interest.

\section{Funding Information}

No sources of funding were declared for this study.

\section{References}

1. Sadock BJ, Sadock VA, Ruiz P (2015) Panic disorder. In: Kaplan and Sadock's Synopsis of Psychiatry $11^{\text {th }}$ edition. Wolters Kluwer Lippincott Williams and Wilkins p: 646.

2. Sarris J, Sinclair J, Karamacoska D, Davidson M, Firth J (2020) Medicinal cannabis for psychiatric disorders: a clinically-focused systematic review. BMC Psychiatry 20: 1-14.

3. Ashton $\mathrm{CH}$ (1999) Adverse effects of cannabis and cannabinoids. $\mathrm{Br}$ J Anaesth 83: 637-649.

4. Shakya DR, Upadhaya SR, Neupane H, Subedi R (2021) Considerations for the Use of Medical Cannabis: An Overview of Benefits and Harms. Biomed J Sci \& Tech Res 36: 28746-28753.

5. Shakya DR, Upadhaya SR (2021) Cannabis Induced Psychotic Disorder in Cannabis Withdrawal during COVID-19 Lockdown: A Case Report. Indian Journal of Clinical Psychiatry 1: 65-68.

6. Shakya DR, Mishra DR, Gyawali R, Rimal SP, Lama S, et al. (2020) COVID-19 Pandemic and BPKIHS: our Situation, Endeavors and Future Direction. JBPKIHS 3: 39-49.

7. Khan MA, Akella S (2009) Cannabis-induced bipolar disorder with psychotic features: a case report. Psychiatry (Edgmont) 6: 44-48.

8. Hall W, Degenhardt L (2000) Cannabis use and psychosis: a review of clinical and epidemiological evidence. Aust N Z J Psychiatry 34: 26-34.

9. Sharma BP (1975) Cannabis and its users in Nepal. Br J Psychiatry 127: 550-552.

10. Prochaska JJ, Das S, Young-Wolff KC (2017) Smoking, Mental Illness, and Public Health. Annu Rev Public Health 38: 165-185. 